\title{
Validation of the Raw National Aeronautics and Space Administration Task Load Index (NASA-TLX) Questionnaire to Assess Perceived Workload in Patient Monitoring Tasks: Pooled Analysis Study Using Mixed Models
}

Sadiq Said ${ }^{1 *}$; Malgorzata Gozdzik ${ }^{*}$; Tadzio Raoul Roche ${ }^{1}$; Julia Braun ${ }^{2}$, PhD; Julian Rössler ${ }^{1}$; Alexander Kaserer ${ }^{1}$, MD; Donat R Spahn ${ }^{1}, \mathrm{MD}$; Christoph B Nöthiger ${ }^{1}, \mathrm{MD}$; David Werner Tscholl ${ }^{1}$, MD

\footnotetext{
${ }^{1}$ Department of Anesthesiology, University Hospital Zurich, Zurich, Switzerland

${ }^{2}$ Department of Epidemiology and Biostatistics, University of Zurich, Zurich, Switzerland

*these authors contributed equally
}

\section{Corresponding Author:}

Sadiq Said

Department of Anesthesiology

University Hospital Zurich

Raemistrasse 100

Zurich, 8091

Switzerland

Phone: 41786718911

Email: sadiq.said@usz.ch

\begin{abstract}
Background: Patient monitoring is indispensable in any operating room to follow the patient's current health state based on measured physiological parameters. Reducing workload helps to free cognitive resources and thus influences human performance, which ultimately improves the quality of care. Among the many methods available to assess perceived workload, the National Aeronautics and Space Administration Task Load Index (NASA-TLX) provides the most widely accepted tool. However, only few studies have investigated the validity of the NASA-TLX in the health care sector.

Objective: This study aimed to validate a modified version of the raw NASA-TLX in patient monitoring tasks by investigating its correspondence with expected lower and higher workload situations and its robustness against nonworkload-related covariates. This defines criterion validity.

Methods: In this pooled analysis, we evaluated raw NASA-TLX scores collected after performing patient monitoring tasks in four different investigator-initiated, computer-based, prospective, multicenter studies. All of them were conducted in three hospitals with a high standard of care in central Europe. In these already published studies, we compared conventional patient monitoring with two newly developed situation awareness-oriented monitoring technologies called Visual Patient and Visual Clot. The participants were resident and staff anesthesia and intensive care physicians, and nurse anesthetists with completed specialization qualification. We analyzed the raw NASA-TLX scores by fitting mixed linear regression models and univariate models with different covariates.
\end{abstract}

Results: We assessed a total of 1160 raw NASA-TLX questionnaires after performing specific patient monitoring tasks. Good test performance and higher self-rated diagnostic confidence correlated significantly with lower raw NASA-TLX scores and the subscores (all $P<.001)$. Staff physicians rated significantly lower workload scores than residents $(P=.001)$, whereas nurse anesthetists did not show any difference in the same comparison $(P=.83)$. Standardized distraction resulted in higher rated total raw NASA-TLX scores $(P<.001)$ and subscores. There was no gender difference regarding perceived workload $(P=.26)$. The new visualization technologies Visual Patient and Visual Clot resulted in significantly lower total raw NASA-TLX scores and all subscores, including high self-rated performance, when compared with conventional monitoring (all $P<.001$ ).

Conclusions: This study validated a modified raw NASA-TLX questionnaire for patient monitoring tasks. The scores obtained correctly represented the assumed influences of the examined covariates on the perceived workload. We reported high criterion validity. The NASA-TLX questionnaire appears to be a reliable tool for measuring subjective workload. Further research should focus on its applicability in a clinical setting. 
(J Med Internet Res 2020;22(9):e19472) doi: 10.2196/19472

\section{KEYWORDS}

workload; questionnaires; National Aeronautics and Space Administration Task Load Index; awareness; situation awareness; patient monitoring; thromboelastometry

\section{Introduction}

\section{Workload}

The World Health Organization considers attentive anesthesia providers to be essential to prevent perioperative disability and death [1]. To maintain high quality of care, all factors negatively affecting human performance should be minimized. Various subjective factors, such as high complexity of tasks, stressful personal factors, high-pressure working environment, lack of situation awareness, fatigue, and increased workload, all impair human performance, the quality of care, and thus patient safety [2-4]. The International Organization for Standardization defines workload as the totality of external conditions and requirements in a work system, which affects the physiological and/or psychological state of a person [5]. The perceived workload and a person's ability to create and maintain adequate situation awareness are interconnected [6]. Situation awareness incorporates the perception of the current status of a situation's critical elements, with understanding of their meaning, and the projection of this knowledge into the near future [2,7]. For physicians and nurses working inside the operating theatre or intensive care unit, it is crucial to keep situation awareness at a high level through constant mental reassessment. This process however requires substantial cognitive effort. A high workload is a psychological stress factor that takes up part of a person's naturally limited working memory and ultimately leads to fewer cognitive resources being available.

Hence, accurate assessment of workload is of great importance to manage stressors. Various methods for quantifying perceived workload have been described, which can be divided into the following two large groups: subjective assessment through questionnaires and objective physiological assessment of variables such as heart rate, galvanic skin resistance, breathing rate, pupil diameter, and blinking frequency [8,9].

\section{National Aeronautics and Space Administration Task Load Index}

The National Aeronautics and Space Administration Task Load Index (NASA-TLX) provides the most widely accepted and validated tool to measure overall workload after completing a task [10-12]. It was initially created by the Human Performance Research Group at NASA Ames Research Center for the aviation industry. Since then, its use has expanded to many other fields such as computer science [13], psychophysiology [14], and transportation [15]. The NASA-TLX is a multidimensional tool that contains six predefined dimensions. Three dimensions measure the demands imposed on the subject (mental, physical, and temporal demands), and three dimensions focus on how the subject deals with the task at hand (self-rated performance, effort, and frustration level). Dividing the workload into six subcategories intends to reduce variability among subjects and show the source of the workload. There are two different methods of using the NASA-TLX tool. The weighted NASA-TLX score is a two-step process, in which the user first rates all six subcategories after completing a specific task and then weights the contribution of each factor in a predefined manner. This aims to further understand which potential source accounts mostly for the perceived workload. On the other hand, in the raw NASA-TLX score, the user rates all six subcategories after completing a specific task, without weighing them. The result is the arithmetic mean of all subscales. Research has shown that the raw NASA-TLX has a high correlation with the weighted one [11], but is more time efficient and simpler to apply [16,17]. We used the raw NASA-TLX questionnaire in several studies within the scope of our research activities in the field of patient monitoring and situation awareness-oriented visualization technologies [18].

\section{Patient Monitoring Tasks}

Patient monitoring can be generalized as continuous observation of a condition or certain parameters, regardless of the method used [19,20]. In previous studies, we simplified the presentation of information in different patient monitoring devices by creating two new situation awareness-oriented information transfer technologies called Visual Patient [21,22] and Visual Clot [23]. We further discuss the functionality and applicability of these technologies in the methods section. In tested scenarios, both Visual Patient and Visual Clot helped the health care provider to make correct diagnoses faster and with less perceived cognitive workload compared with standard presentation only.

\section{Study Aims and Hypotheses}

Only few studies have investigated the validity of the raw NASA-TLX score in the health care sector [24-27]. There is no existing gold standard in measuring workload. The primary objective of this study was to validate the raw NASA-TLX questionnaire in patient monitoring tasks by investigating a broad set of over 1000 NASA-TLX scores. We based our evaluation of the robustness of the questionnaire against covariates not associated with workload and on the relation of the NASA-TLX scores with covariates that are known to influence workload. Therefore, we investigated criterion validity. We expect that the scores will increase with the difficulty of the task and distraction, and decrease with the work experience and self-confidence of the user. Further, we assume that the new visualization technologies will reduce all dimensions of the workload approximately uniformly and that not one workload aspect alone will be responsible for observed reductions.

\section{Methods}

\section{Ethical Permission}

The leading ethics committee (Cantonal Ethics Committee of Zurich, Switzerland) reviewed the study protocols for all four 
included studies and issued a declaration of no objection for each one of them (reference numbers: 2016-00103, 2017-00795, and 2018-00933). Reference number 2017-00795 covers two of the studies, as they were performed simultaneously and included the same participants. Additionally, before participation in this study, we obtained written informed consent from all participants to collect data for scientific purposes and publications.

\section{Study Design and Data Collection}

To validate the NASA-TLX, we analyzed data from four different studies all performed by anesthesia and intensive care providers at the University Hospital Zurich and Cantonal
Hospital Winterthur in Switzerland, and University Hospital Frankfurt in Germany [21,23,28,29]. Table 1 provides an overview of the included studies. All of them were investigator-initiated, computer-based, prospective, dual-center studies, and have been published in the past 2 years [21,23,28,29]. In all studies, we based the recruitment of participants on their clinical availability. The day before data collection, we contacted available individuals by institutional email or telephone to plan participation in the study. We then carried out data collection during regular working hours. During this time, we released them from all other duties, including telephone availability.

Table 1. Description of the four studies used with the respective participant numbers and completed National Aeronautics and Space Administration Task Load Index questionnaires.

\begin{tabular}{|c|c|c|c|}
\hline Study title ${ }^{\mathrm{a}}$ & Location & Number of participants & NASA-TLX ${ }^{\mathrm{b}}$ questionnaires, $\mathrm{n}$ \\
\hline $\begin{array}{l}\text { Using an Animated Patient Avatar to Improve Perception of Vital } \\
\text { Sign Information by Anesthesia Professionals }\end{array}$ & $\mathrm{USZ}^{\mathrm{c}}$ and $\mathrm{KSW}^{\mathrm{d}}$ & 32 & 128 \\
\hline $\begin{array}{l}\text { Avatar-Based Versus Conventional Vital Sign Display in a Central } \\
\text { Monitor for Monitoring Multiple Patients: A Multicenter Computer- } \\
\text { Based Laboratory Study }\end{array}$ & USZ and KSW & 38 & 312 \\
\hline $\begin{array}{l}\text { Effects of a Standardized Distraction on Caregivers' Perceptive } \\
\text { Performance with Avatar-Based and Conventional Patient Moni- } \\
\text { toring: A Multicenter Comparative Study }\end{array}$ & USZ and KSW & 38 & 312 \\
\hline $\begin{array}{l}\text { Improving Decision Making Through Presentation of Viscoelastic } \\
\text { Tests as 3D Animated Blood Clot: the Visual Clot }\end{array}$ & USZ and $\mathrm{UKF}^{\mathrm{e}}$ & 60 & 720 \\
\hline
\end{tabular}

${ }^{\mathrm{a}}$ The second and third studies included the same participants.

bASA-TLX: National Aeronautics and Space Administration-Task Load Index.

${ }^{\mathrm{c}}$ USZ: University Hospital Zurich.

${ }^{\mathrm{d}} \mathrm{KSW}$ : Cantonal Hospital of Winterthur.

${ }^{\mathrm{e}}$ UKF: University Hospital Frankfurt.

In all included studies, we used the original formulation of the NASA-TLX questionnaire provided by the official NASA website. However, we modified the raw NASA-TLX surveys from six to only five dimensions by removing the physical demand question as our tasks did not require any physical effort. Table 2 shows the modified raw NASA-TLX questionnaire we used in our studies. Participants were staff or resident anesthesiologists and nurse anesthetists with completed specialization qualification. They were all employed in the abovementioned centers during the course of the trials. We selected the participants randomly, regardless of sex, age, job description, staff position, or education level. Participation was voluntary, and none of the subjects received compensation in any form.

Table 2. Description of the modified raw National Aeronautics and Space Administration Task Load Index questions and rating scale.

\begin{tabular}{lll}
\hline Workload & Descriptive question & Endpoint $^{\mathrm{a}}$ \\
\hline Mental demand & Was the task easy or demanding, simple or complex? & 0 to 100 \\
Temporal demand & How much time pressure did you feel performing the task? & 0 to 100 \\
Self-rated performance & How successful or satisfied did you feel upon the performance or completion of the & 0 to 100 \\
Effort & $\begin{array}{l}\text { given task? } \\
\text { How hard did you have to work (mentally and physically) to accomplish your level } \\
\text { of performance? }\end{array}$ & 0 to 100 \\
Frustration level & $\begin{array}{l}\text { How insecure, discouraged, stressed, and annoyed versus content, relaxed, and } \\
\text { complacent did you feel during the task? }\end{array}$ & 0 to 100 \\
\hline
\end{tabular}

${ }^{\text {a }}$ Subjects rated the subscores numerically from 0 (very low) to 100 (very high). The endpoints regarding performance are inverted with 0 indicating very good performance and 100 indicating very poor performance.

${ }^{\mathrm{b}}$ The term self-rated performance indicates the performance dimension of the National Aeronautics and Space Administration Task Load Index score. 


\section{Visual Patient and Visual Clot Technologies}

Visual Patient technology is a situation awareness-oriented visualization tool that displays up to 11 of the most frequently used patient vital signs in the form of a patient avatar in addition to the standard monitoring screen [21,22]. Visual Patient transforms the conventional numerical and waveform display of the vital signs in real time into a patient avatar with the ability to adjust its color, shapes, and rhythmic movements depending on the patient's current situation. For example, if a patient shows an elevated body temperature (ie, more than $37.5^{\circ} \mathrm{C}$ ), the avatar will display heat radiation rising from the patient model. Another example is the patient's neuromuscular state of relaxation, which is best described by the train-of-four ratio. If the mentioned ratio drops below 20 , the patient avatar changes its posture and goes into a floppy state. In situations where several vital signs are out of range, Visual Patient technology is able to illustrate them simultaneously. Visual Clot technology [23] on the other hand illustrates abstract conventional viscoelastic rotational thromboelastometry (ROTEM) readings in form of a three-dimensional animated blood clot. This aims to help the user create a simpler mental model of the current coagulation disorder. The image consists of a blood clot model with its various components such as platelets, coagulation factors, and enzymes. Based on established conventional monitoring values, the visualization shows these coagulation components as either present or not. Figure 1 illustrates both the Visual Clot and Visual Patient technologies. We have provided instructional videos in Multimedia Appendix 1 and Multimedia Appendix 2 explaining how both technologies work.

Figure 1. Graphic showing the Visual Clot and Visual Patient technologies. (A) Bleeding Visual Clot illustrated with its different coagulation components. These are either present or absent, depending on the coagulation status. (B) Visual Patient with its different visualizations of vital parameters.

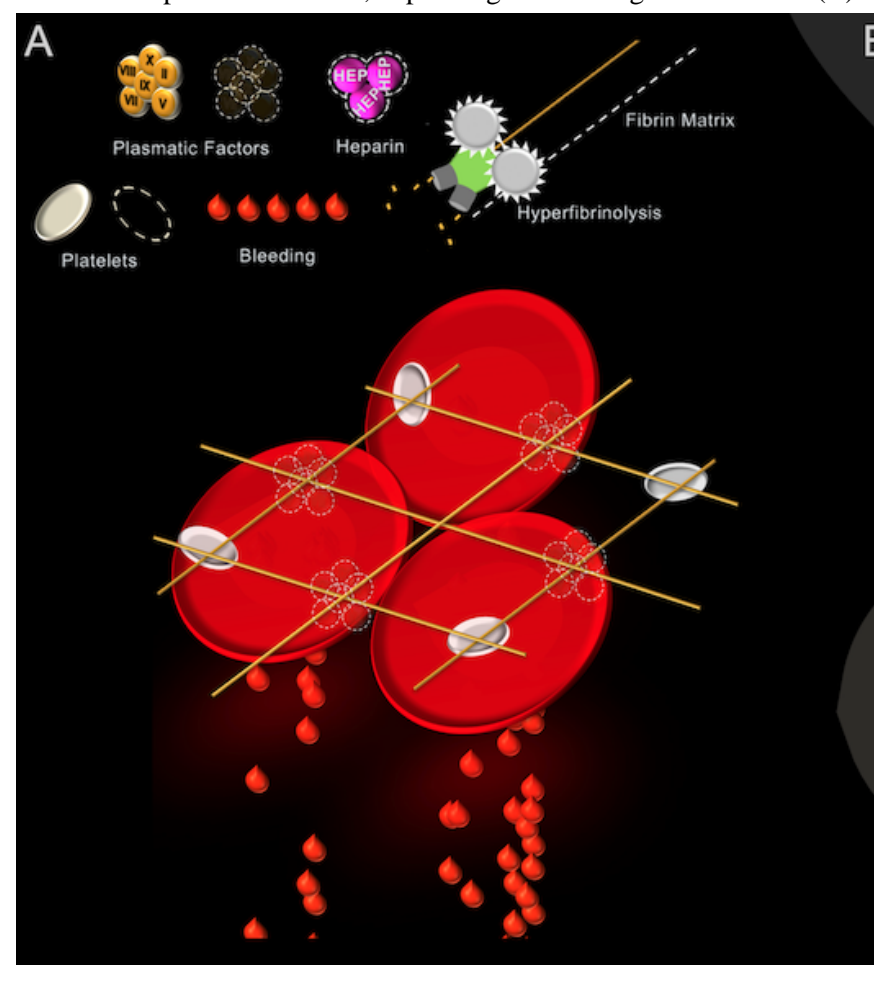

A relevant limitation for both visualization technologies is the absence of quantification values. Both avatars only display the following three states: too low, within range, and too high. This limitation defines the intended use of the technologies, which is the situation awareness-oriented supplementation of conventional monitoring methods. They were not designed to replace numerical values. Both visualization technologies were invented, prototyped, and patented by our research group at the Institute for Anesthesiology of the University Hospital Zurich. We are developing Visual Patient into a product under a Joint Development and Licensing Agreement with Royal Philips NV and Philips Medizin-Systeme Böblingen GmBH. Regarding Visual Clot, we have signed a letter of intent with Instrumentation Laboratory. Both technologies are in the prototype stage of development and neither Visual Patient nor Visual Clot is currently CE certified as a medical device.

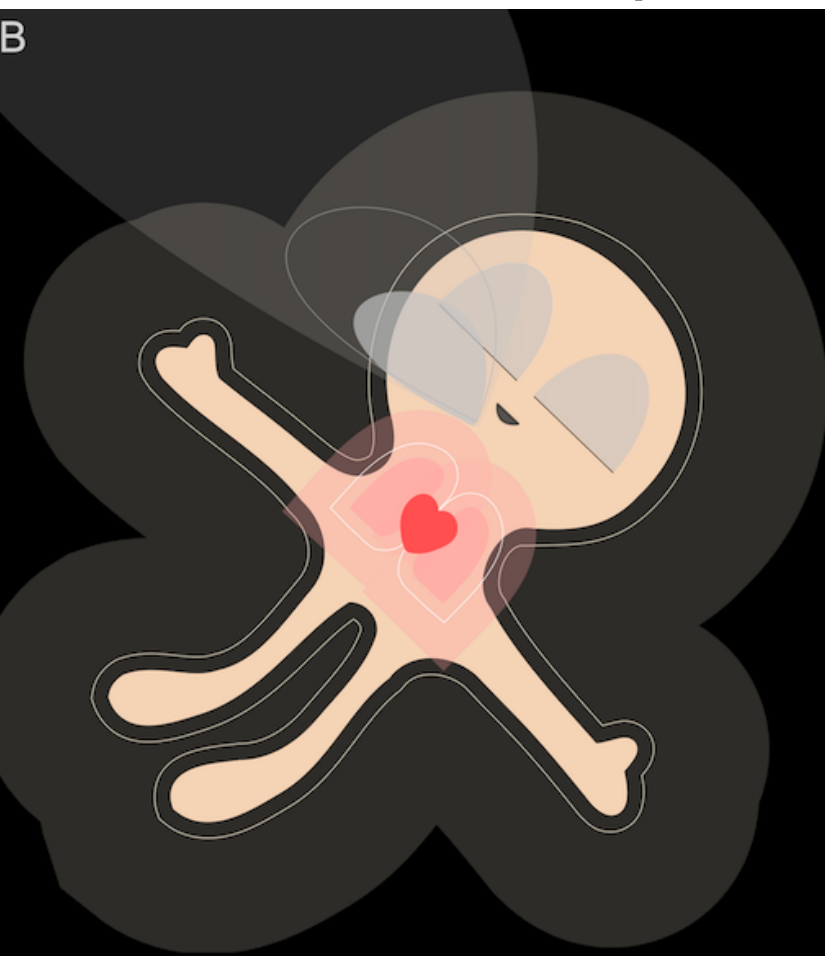

\section{Patient Monitoring Tasks}

We assessed the perceived workload using the raw NASA-TLX questionnaire after performing patient monitoring tasks using either one of the newly developed visualization tools (Visual Patient or Visual Clot) or respective conventional monitoring alone. The monitoring scenarios appeared in randomized order for predefined relatively short periods. Furthermore, the participants had to rate their diagnostic confidence level on a four-point Likert scale ranging from "very unconfident" to "very confident" to further assess uncertainty as a psychological stress factor. In the four mentioned studies, we generated workload through specific tasks as follows:

- In the primary Visual Patient study [21], we displayed four patient monitoring scenarios on a computer for 3 or 10 seconds, portraying either the conventional monitoring screen or the animated patient avatar in randomized order. 
Subsequently, the participants had to recall the patient's condition.

- The Visual Patient central monitoring study [29] included four different scenarios in which we showed two critical and four healthy patients simultaneously on a central monitor (as in the intensive care unit or operating room) for 10 or 30 seconds. Afterwards, the participants had to recall the patient's condition.

- The same participants (as in the central monitoring study [29]) also took part in the Visual Patient distraction study [28]. In this study, we distracted the participants with standardized simple calculation tasks. Simultaneously, we showed them different monitoring scenarios either with the conventional monitoring screen alone or with the help of Visual Patient. The workload task also demanded recall of the patient's condition.

- Finally, in the Visual Clot study [23], we showed the participants 12 scenarios representing six different hemostatic conditions, which they had to solve using either standard viscoelastic ROTEM results alone or using the matching animated blood clot.

\section{Validation Method and Studied Covariates}

We investigated the robustness of the NASA-TLX questionnaire based on criterion validity, which can be further divided into predictive and concurrent validity. Predictive validity indicates the extent to which an assumption under investigation can be predicted [30]. Concurrent validity compares the result in question with an already known relationship of the same variable [30].

In all included studies, we had consistently recorded 11 different covariates. In this pooled analysis, we determined the expected impact on workload from these measured covariates, using both literature research and logical deductions. It was described that more professional experience should result in lower cognitive workload [31,32]. Since we had recorded the educational stage of the participants through their job descriptions, we regarded this equal to experience. Further, we correlated the measured covariate self-rated confidence with experience and test performance through both literature research and logical reasoning. In order to not confuse the terms, in this manuscript, we defined test performance as the actual testing outcome of the participants and self-rated performance as the subscores of the NASA-TLX. We expected a decreased NASA-TLX score in participants with high self-confidence. Regarding the actual test performance, we investigated predictive validity. We expected good performing participants to perceive less workload. As far as the task's difficulty is concerned, it cannot be assessed directly as various factors influence it. We defined scenarios in which distraction occurred as more difficult. They divide one's attention and thus affect the work-related receptiveness [33]. Therefore, we expected the results to show an increased perceived workload and thus correspond to concurrent validity. We did not find any evidence in a literature search and everyday clinical practice of a different perceived workload between the sexes. Therefore, we regarded this as a covariate without an expected influence on workload.

\section{Statistical Analysis}

We validated the raw NASA-TLX score and the different subscores by fitting mixed linear regression models with a random intercept per person (to cover repeated measurements) and a random intercept per study. We fitted univariate models with binary test performance (task correct or incorrect), binary confidence (unconfident or confident), distraction, center, gender, profession, binary daytime (above or below the median), binary playback sequence (first or second half of the tasks), and central monitor performance as covariates.

We analyzed interactions of some covariates with the technology variable to explore more in depth why the NASA-TLX and its subscores improved in the case of the new visual technologies. To explore which subscore benefited the most from the new visualization technologies, we calculated univariate models for each subscore that included only the technology variable, and we compared the size of the estimated coefficients. To characterize the individuals who benefited the most from the introduction of the new technologies, we fitted a joint model for the total NASA-TLX with the technology variable and several other covariates. In one additional model per variable, we included an interaction term between technology and the respective covariate to see if the impact of certain variables was particularly strong in the case of the new technologies.

All analyses were performed using $\mathrm{R}$ version 3.6.2 ( $\mathrm{R}$ Foundation for Statistical Computing). We considered a $P$ value $<.05$ to indicate statistical significance.

\section{Data Sharing Statement}

We provide the complete data used for this study in Multimedia Appendix 3.

\section{Results}

\section{Study and Participant Characteristics}

In all four evaluated studies [21,23,28,29], 128 anesthesia providers participated and rated a total of 1160 NASA-TLX questionnaires. Overall, 552 of 1160 (47.6\%) NASA-TLX surveys were collected at the University Hospital Zurich, 360 of $1160(31.0 \%)$ at the University Hospital Frankfurt, and 248 of $1160(21.4 \%)$ at the Cantonal Hospital Winterthur. Further, 648 of $1160(55.9 \%)$ ratings were provided by male participants, and 512 of $1160(44.1 \%)$ by female participants. According to job description, 556 of 1160 (47.9\%) ratings were provided by staff physicians, 432 of 1160 (37.2\%) by resident physicians, and 172 of $1160(14.8 \%)$ by nurse anesthetists. Comparing the technologies used, $62.1 \%$ (720/1160) of all data originated from the three Visual Patient projects, and $37.9 \%$ (440/1160) from the Visual Clot study.

\section{Quantitative Analyses of the NASA-TLX Questionnaire}

In Figure 2 and Figure 3, we illustrate the correlation of the total NASA-TLX workload score and its subscores after performing univariate analysis with test performance, confidence, distraction, center, gender, job description, daytime, and playback sequence as different covariates. The playback sequence was described as the first or second half of the task. We provide the NASA-TLX coefficient and the 95\% CI. 
Figure 2. Correlation of different covariates with the total score of the National Aeronautics and Space Administration Task Load Index (NASA-TLX) workload assessment tool. Left and right of the dashed line indicate lower and higher perceived workload, respectively. KSW: Cantonal Hospital Winterthur; UKF: University Hospital Frankfurt.

\section{Validation of the total NASA-TLX score}

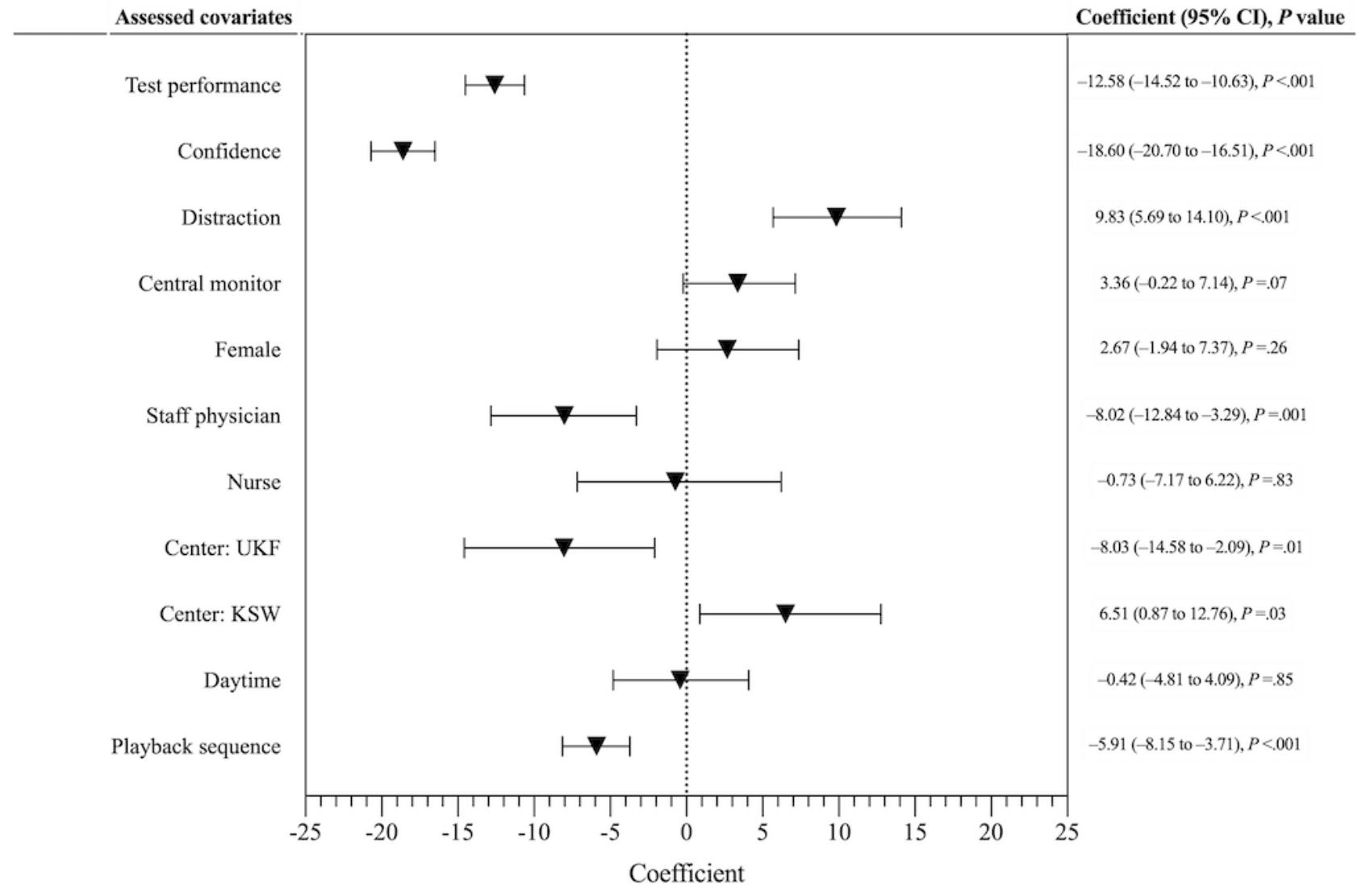

The total workload score analysis showed that participants' test performance and higher confidence correlated significantly with lower NASA-TLX scores (both $P<.001$ ). Compared with the University Hospital Zurich, participants from the University Hospital Frankfurt had significantly lower total workload scores $(-8.03,95 \%$ CI -14.58 to $-2.09 ; P=.01)$. Further, the second half of the playback sequence had a significant positive effect on lowering the perceived workload $(-5.91,95 \% \mathrm{CI}-8.15$ to $-3.71 ; P<.001)$. Regarding the job position, resident physicians served as the comparison. Staff physicians $(-8.02,95 \% \mathrm{CI}$ -12.84 to $-3.29 ; P=.001)$ rated significantly lower workload than residents, whereas nurses did not show any significant difference compared with residents $(P=.83)$. Distraction and the Cantonal Hospital Winterthur compared with the University Hospital Zurich correlated significantly with higher rated NASA-TLX scores $(P<.001$ and $P=.03$, respectively). The other listed covariates did not show any relevant difference.

Figure 3 illustrates the entire evaluation of the examined covariates for the subscores of the NASA-TLX. Good test performance and high confidence level after performing the task correlated significantly with lower workload scores in every subcategory of the NASA-TLX (all $P<.001$ ). Staff physicians also differed significantly from resident physicians with lower workload scores in every subcategory, except the frustration level $(-6.93,95 \% \mathrm{CI}-13.68$ to $0.01 ; P=.05)$. Distraction was the only covariate to show a relevant effect on increasing the workload in every subscore of the NASA-TLX. Comparing the participants at the different centers with those at the University Hospital Zurich, the participants at the University Hospital Frankfurt had less mental $(P=.02)$ and temporal $(P=.002)$ demands, and less perceived effort $(P=.003)$. The participants at the Cantonal Hospital Winterthur had more mental $(P=.007)$ and temporal demands $(P=.02)$, and more required effort $(P=.03)$. We observed no gender difference in all subscores. Showing several patients simultaneously on a central monitor correlated significantly with increased mental demand $(P=.05)$ and increased effort $(P=.01)$ to fulfill the task. We observed no effect for temporal demand $(0.77,95 \% \mathrm{CI}-3.34$ to $4.91 ; P=.72)$, frustration level $(3.10,95 \% \mathrm{CI}-1.43$ to $7.43 ; P=.17)$, and self-rated performance $(2.04,95 \% \mathrm{CI}-2.76$ to $7.20 ; P=.42)$ using a central monitor. Playback sequence showed its only significant effect on the temporal demand subscore with lower perceived workload in the second half of the task $(-3.24,95 \%$ CI -5.78 to $-0.74 ; P=.01)$. 
Figure 3. Correlation of different covariates with subscores of the National Aeronautics and Space Administration Task Load Index (NASA-TLX) workload assessment tool. The original NASA-TLX questionnaire evaluated performance on an inverted X-axis from perfect to failure, with a low raw score corresponding to good self-rated performance. Therefore, to ensure that the X-axis in this figure is the same, the self-rated performance is displayed inverted. KSW: Cantonal Hospital Winterthur; UKF: University Hospital Frankfurt.

\section{Validation of NASA-TLX sub scores}

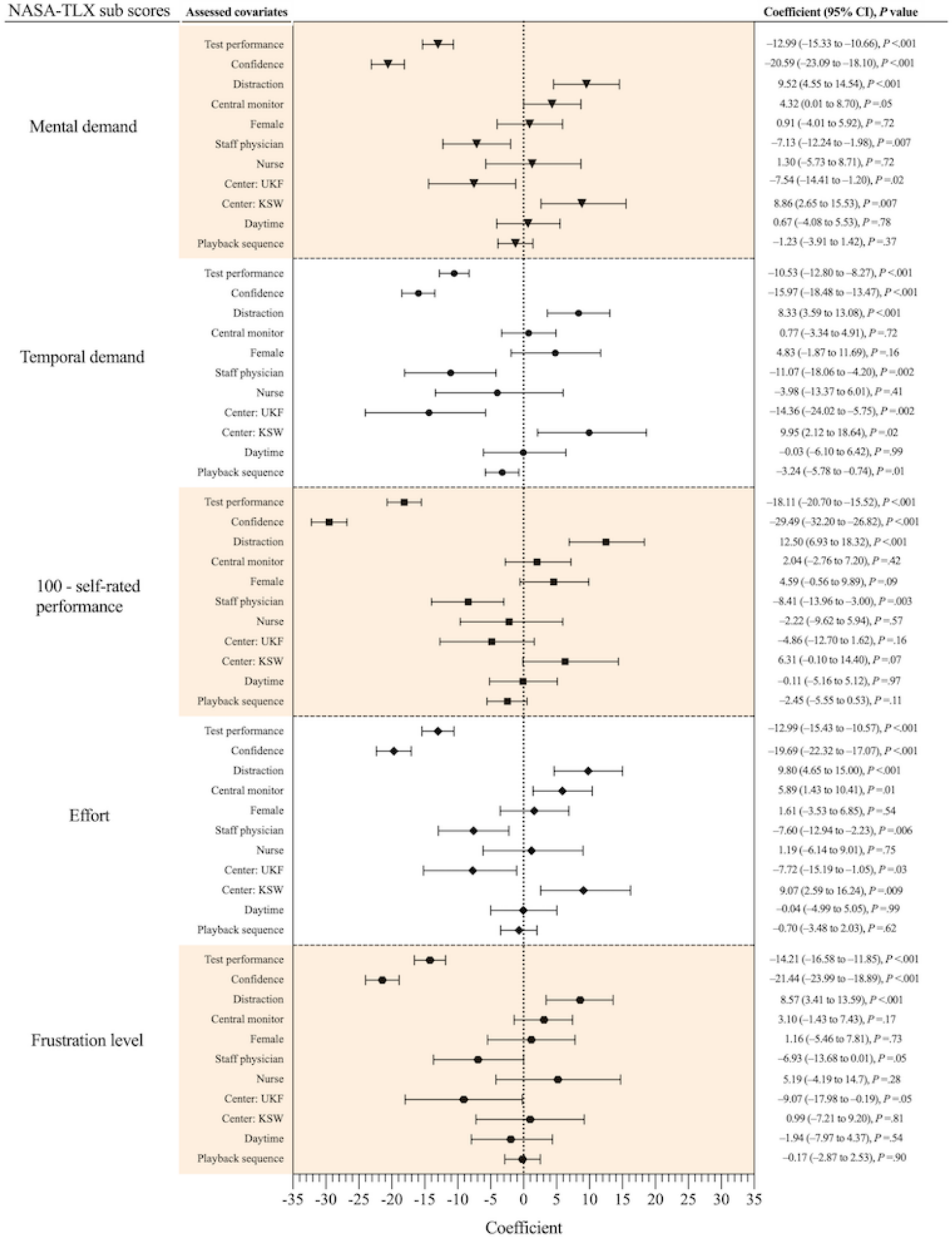

Performing the same tasks, we compared the conventional monitoring devices with the respective visualization technologies. The total NASA-TLX score and all listed subscores correlated significantly with lower workload scores when using Visual Patient and Visual Clot (all $P<.001$ ). Table
3 demonstrates the comparison of the new visualization technologies with respective conventional monitoring. The self-rated performance dimension showed the most relevant change in the perceived workload assessment (coefficient -18.28). 
Table 3. Overall comparison of the new visual technologies Visual Patient and Visual Clot with conventional monitoring.

\begin{tabular}{|c|c|c|c|c|}
\hline Variable & Coefficient & CI lower & CI upper & $P$ value \\
\hline Total & -14.36 & -16.06 & -12.65 & $<.001$ \\
\hline Mental demand & -15.79 & -17.85 & -13.72 & $<.001$ \\
\hline Temporal demand & -10.53 & -12.59 & -8.46 & $<.001$ \\
\hline Self-rated performance ${ }^{a}$ & -18.28 & -20.63 & -15.93 & $<.001$ \\
\hline Effort & -16.80 & -18.92 & -14.68 & $<.001$ \\
\hline Frustration level & -15.97 & -18.06 & -13.87 & $<.001$ \\
\hline
\end{tabular}

${ }^{\mathrm{a}}$ The term self-rated performance indicates the performance dimension of the National Aeronautics and Space Administration Task Load Index score.

\section{Data Sharing Statement}

We provide the complete analysis of used data for this study in Multimedia Appendix 4.

\section{Discussion}

\section{Principal Findings}

This pooled analysis examined a broad set of raw NASA-TLX scores obtained after performing patient monitoring tasks. Good test performance, high self-confidence in successfully completing a task, high hierarchical job position, and training correlated with decreased raw NASA-TLX workload scores, whereas distraction scenarios increased perceived workload. The questionnaire was robust against nonworkload-related factors such as gender. The new visualization technologies Visual Patient and Visual Clot both decreased all workload dimensions compared with conventional monitoring alone and patient monitoring reference values provided by the literature [11]. Reducing workload helps to free cognitive resources, which can be used to understand the provided monitoring information crucial to maintain a high quality of care [7].

The analysis of 1160 raw NASA-TLX questionnaires showed that self-rated high confidence correlated significantly with lower overall raw NASA-TLX scores and its subscores (all $P<.001)$. Furthermore, staff physicians had significantly lower total workload scores compared with residents. During clinical residency training, inexperienced physicians go through a period of professional and personal growth. They acquire knowledge and skills [6], and experience many challenging clinical situations that build professional competency and thereby increase their confidence at work [34]. Generally, staff physicians are more certain of their clinical abilities and thus exude more confidence than residents. The participants at the University Hospital Frankfurt also rated lower total workload scores compared with those at the University Hospital Zurich. This is in line with our abovementioned train of thought, as the participating physicians in Frankfurt had more professional experience overall than those in Zurich [23]. When comparing nurses with residents, there was no difference in the perceived total workload after performing the same monitoring task. We explain this interesting finding on the basis of existing practical experience. All nurses who took part in this study had completed their specialty qualification and thus often had more professional experience than the residents who were still in training. Their self-confidence may have increased owing to their completed education. Our results confirm our hypothesis that confidence as a trait, which is compatible with a higher job position, lowers perceived workload. It is known from other domains that individuals with higher confidence make better decisions [35]. The World Health Organization aims to continuously improve, protect, and promote the health, safety, and well-being of all workers [36]. Analyzing workload in the perioperative setting can lead directly to practical work-based implications, such as tailored task assignment and improved training plans, for residents or other personnel. This efficient identification and management of workload influences employee well-being [37] and lowers the source of fatigue from work overload, which is an independent risk factor for exhaustion and burnout [38]. Moreover, workload has been shown to be associated with adverse patient outcomes [39-41].

Good test performance in conducting patient monitoring tasks correlated with lower total raw NASA-TLX scores, as well as all subscores, including high self-rated performance. We propose that this connection resulted from both training and experience. When a person has received a lot of training and thus experience in performing a task, the perceived workload decreases and the actual performance increases. Nevertheless, this finding allows not drawing a linear relationship between task performance and workload. This relation is complex when investigated in more detail. Both excessive workload and low demand situations can degrade performance [42], and additional factors, such as personal resilience, influence the work capacity to a great extent. This shows that there are other factors apart from workload that influence task performance.

Further, our results showed that standardized distraction negatively affected the raw NASA-TLX scores with all subcategories. This is in line with our hypothesis that distractions increase perceived workload. They take up part of the already limited mind while coping with several things simultaneously and reduce the work-related memory capacity of humans. It was shown that distractions impair situational awareness and thus affect clinical decision making [33,43]. The source of most anesthesia adverse events lies in reduced situational awareness [4,44]. This further demonstrates the importance of minimizing working environment distractions in areas that involve workload-sensitive tasks such as patient monitoring inside the operating theatre [45]. In one study, 22 of $25(88 \%)$ anesthesia providers agreed to the statement that human factor problems do lead to critical information not being received [46]. 
The newly developed visualization technologies Visual Patient and Visual Clot were associated with decreased perceived workload as expected. These technologies have been developed to link several sources of information together and create an avatar-based visualization aimed to facilitate the mental model of the current situation [21-23]. Endsley defined the goal of optimal situation awareness-oriented design to transfer information as quickly as possible and with the least cognitive effort [7].

Decreasing workload while monitoring a patient reduces psychological stress by saving cognitive resources, which are especially needed in critical situations. In 2015, Grier et al [11] examined a vast amount of published NASA-TLX scores in a meta-analysis showing the distribution frequency of the scores by task type. They analyzed 174 monitoring-type tasks involving change detection, speech detection, and vigilance tasks. A mean NASA-TLX global workload score of 52.24 with an IQR of 22.66 (39.97-62.63) was reported [11]. This fits the mean NASA-TLX global workload score of 54.60 with an IQR of 27.0 (42.0-69.0), which we observed when evaluating our conventional monitoring tasks. Using the situation awareness-oriented visualization monitoring technologies, we found a mean NASA-TLX global workload score of 40.2 with an IQR of 31.0 (25.0-56.0). These technologies lowered the mean perceived workload compared with the literature value by $23.0 \%$. This is comparable to a workload that occurs when driving a car (mean 41.52, IQR 23.7 [28.05-51.73]) [11].

This study contributes to the ongoing validation of the NASA-TLX in the medical field. The results are consistent with those of other studies that have found high construct validity of the NASA-TLX score [24,25]. These studies described an increase in the workload score due to distraction [47], case complexity [24], and low task performance [48], and a reduction due to training [49]. Other studies found good correlation with other questionnaires for workload assessment [50,51] and with physiological stress measurements [52,53].

\section{Strengths and Limitations}

Our study had several limitations. All tasks used to validate the raw NASA-TLX questionnaire took place in a testing environment with clearly defined monitoring limits within scenarios. Perceived workload and therefore its assessment might differ in a clinical setting, where each situation must be interpreted independently. Future studies should test the applicability of the raw NASA-TLX in the clinical setting. However, it is plausible that this effect is marginal as the score reflects a subjective evaluation while information intake and thus perception of workload remain similar. Further, we assessed and validated a modified version of the questionnaire. Since our monitoring tasks did not require any relevant physical effort, we removed this dimension from the scale in order not to distort the value of the total workload assessed. Future studies are required to investigate whether such a modification affects the internal consistency of the NASA-TLX questionnaire. Moreover, a true validation would correlate obtained scores with objectively measurable stress characteristics such as heart rate and pupil diameter. Another limitation is that all patient monitoring scenarios took place in central Europe in high quality of care hospitals. Perceived workload can differ in other parts of the world and might influence the reproducibility of the assessment. Finally, interpretation of the raw NASA-TLX scores requires comparative values after performing similar tasks. Therefore, more available data using the same questionnaire in patient monitoring tasks would reduce this limitation. Nevertheless, the results of this study support the use of the raw NASA-TLX score in patient monitoring tasks.

Among the particular strengths of this study are the multicenter design, the large data set, and the consistent recording of identical covariates in all included studies. We examined more than 1000 raw NASA-TLX questionnaires, which were completed by 130 participants, with individual selection solely based on daily clinical availability. This large proportion of staff from the respective institutions constitutes a representative sample. Further, in all included studies, intraparticipant comparisons took place as there was an evaluation of the same monitoring task with both examined interface designs (ie, conventional versus visualization). This greatly reduces the influence of confounding variables if the main factor responsible for the difference remains the interface modality shown, and it further increases the quality of the study.

\section{Conclusions}

For patient monitoring, this study validated a modified version of the raw NASA-TLX questionnaire, in which the physical dimension had been removed from the scale owing to the nature of the given tasks. The obtained scores correctly depicted the assumed influences of the covariables that affect perceived workload. This provided a high extent of criterion validity. The modified raw NASA-TLX questionnaire appears to be a reliable tool for measuring the subjective workload of anesthesia providers who monitor patients inside the operating room. Further research is needed to investigate the applicability of the NASA-TLX questionnaire in the clinical setting and its transferability to personnel working in intensive care units. Moreover, a true validation study for the subjective workload assessment should correlate the NASA-TLX scores with objectively measurable stress characteristics such as heart rate and pupil diameter.

\section{Acknowledgments}

The authors are thankful to the study participants for their time and effort. The Institute of Anesthesiology of the University Hospital Zurich, Zurich, Switzerland, funded this study, and DWT received a career development grant from the University of Zurich. 


\section{Authors' Contributions}

SS, GM, TR, DS, CN, and DT helped to design the study; JR, AK, CN, and DT helped to collect the data; SS, JB, and DT helped to analyze the data; and SS, MG, TR, AK, JB, JR, DS, CN, and DT helped to write the manuscript and approved the final version.

\section{Conflicts of Interest}

The University of Zurich owns the intellectual property rights to the technologies described in this manuscript and registered "Visual Clot" and "Visual Patient" as trademarks. The University of Zurich and Instrumentation Laboratory Company/Werfen Corporation, Bedford, MA, USA, signed a letter of intent regarding a proposed joint development and licensing agreement to develop a product based on the concept of Visual Clot. As designated inventors DS, CN, and DT may receive royalties in the event of commercialization. The authors DT, DS, and CN are in a joint development agreement with the monitoring manufacturer Philips Healthcare (Koninklijke Philips NV) for Visual Patient. Within the framework of this cooperation, a monitoring system based on an avatar will be developed. Within the framework of licensing the technology via the University, the authors DT and $\mathrm{CN}$ might receive royalties as designated inventors in the event of successful product release. DS academic department is receiving grant support from the Swiss National Science Foundation, Berne, Switzerland; the Swiss Society of Anesthesiology and Reanimation (SGAR), Berne, Switzerland; the Swiss Foundation for Anesthesia Research, Zurich, Switzerland; and Vifor SA, Villars-sur-Glâne, Switzerland. DS is the co-chair of the ABC-Trauma Faculty, sponsored by unrestricted educational grants from Novo Nordisk Health Care AG, Zurich, Switzerland; CSL Behring GmbH, Marburg, Germany; LFB Biomédicaments, Courtaboeuf Cedex, France; and Octapharma AG, Lachen, Switzerland. Dr Spahn received honoraria/travel support for consulting or lecturing from Danube University of Krems, Austria; US Department of Defense, Washington, USA; European Society of Anesthesiology, Brussels, BE; Korean Society for Patient Blood Management, Seoul, Korea; Korean Society of Anesthesiologists, Seoul, Korea; Network for the Advancement of Patient Blood Management, Haemostasis and Thrombosis, Paris, France; Baxalta Switzerland AG, Volketswil, Switzerland; Bayer AG, Zürich, Switzerland; B. Braun Melsungen AG, Melsungen, Germany; Boehringer Ingelheim GmbH, Basel, Switzerland; Bristol-Myers-Squibb, Rueil-Malmaison Cedex, France and Baar, Switzerland; CSL Behring GmbH, Hattersheim am Main, Germany and Berne, Switzerland; Celgene International II Sàrl, Couvet, Switzerland; Daiichi Sankyo AG, Thalwil, Switzerland; Ethicon Sàrl, Neuchâtel, Switzerland; Haemonetics, Braintree, MA, USA; Instrumentation Laboratory (Werfen), Bedford, MA, USA; LFB Biomédicaments, Courtaboeuf Cedex, France; Merck Sharp \& Dohme, Kenilworth, New Jersey, USA; PAION Deutschland GmbH, Aachen, Germany; Pharmacosmos A/S, Holbaek, Denmark; Photonics Healthcare BV, Utrecht, Netherlands; Pfizer AG, Zürich, Switzerland; Pierre Fabre Pharma, Alschwil, Switzerland; Roche Diagnostics International Ltd, Reinach, Switzerland; Sarstedt AG \& Co, Sevelen, Switzerland and Nümbrecht, Germany; Shire Switzerland GmbH, Zug, Switzerland; Tem International GmbH, Munich, Germany; Vifor Pharma, Munich, Germany, Neuilly sur Seine, France, and Villars-sur-Glâne, Switzerland; Vifor (International) AG, St. Gallen, Switzerland; and Zuellig Pharma Holdings, Singapore, Singapore. CN and DT received travel support for consulting and lecturing from Instrumentation Laboratory (Werfen), Bedford, MA, USA. CN and DT received proof-of-concept funding from the University of Zurich to prototype Visual Patient. The University of Zurich and Koninklijke Philips NV, Amsterdam, Netherlands entered a joint development and licensing agreement to develop a product based on Visual Patient. As inventors, CN and DT may receive royalty payments in the event of commercialization. AK received honoraria for lecturing from Bayer AG (Switzerland). The other authors do not have any conflicts of interest.

\section{Multimedia Appendix 1}

Instructional video explaining the Visual Clot technology.

[MOV File, 13733 KB-Multimedia Appendix 1]

\section{Multimedia Appendix 2}

Instructional video explaining the Visual Patient technology.

[MOV File, 14845 KB-Multimedia Appendix 2]

\section{Multimedia Appendix 3}

Complete data for this study.

[XLSX File (Microsoft Excel File), 5971 KB-Multimedia Appendix 3]

\section{Multimedia Appendix 4}

Complete analysis of used data for this study.

[PDF File (Adobe PDF File), 145 KB-Multimedia Appendix 4]

\section{References}

1. World Health Organization. Safe Surgery Saves Lives. PACEsetterS 2008;5(3):21. [doi: 10.1097/01.jbi.0000393253.18990.c6] 
2. Schulz CM, Endsley MR, Kochs EF, Gelb AW, Wagner KJ. Situation awareness in anesthesia: concept and research. Anesthesiology 2013 Mar;118(3):729-742. [doi: 10.1097/ALN.0b013e318280a40f] [Medline: 23291626]

3. Reason J. Human error: models and management. West J Med 2000 Jun;172(6):393-396 [FREE Full text] [doi: 10.1136/ewjm.172.6.393] [Medline: 10854390 ]

4. Schulz CM, Krautheim V, Hackemann A, Kreuzer M, Kochs EF, Wagner KJ. Situation awareness errors in anesthesia and critical care in 200 cases of a critical incident reporting system. BMC Anesthesiol 2016 Jan 16;16:4. [doi: 10.1186/s12871-016-0172-7] [Medline: 26772179]

5. Nachreiner F. International standards on mental work-load--the ISO 10,075 series. Ind Health 1999 Apr;37(2):125-133 [FREE Full text] [doi: 10.2486/indhealth.37.125] [Medline: 10319562 ]

6. Goldman J, Wong BM. Nothing soft about 'soft skills': core competencies in quality improvement and patient safety education and practice. BMJ Qual Saf 2020 Aug;29(8):619-622. [doi: 10.1136/bmjqs-2019-010512] [Medline: 32066572]

7. Endsley MR. Designing for Situation Awareness An Approach to User-Centered Design, Second Edition. Boca Raton: CRC Press, Inc; Apr 19, 2016.

8. Kremer L, Leeser L, Breil B. Mental Workload Relating Health Information System - A Literature Review. Stud Health Technol Inform 2019 Sep 03;267:289-296. [doi: 10.3233/SHTI190840] [Medline: 31483284]

9. Al Abdi RM, Alhitary AE, Abdul Hay EW, Al-Bashir AK. Objective detection of chronic stress using physiological parameters. Med Biol Eng Comput 2018 Dec;56(12):2273-2286. [doi: 10.1007/s11517-018-1854-8] [Medline: 29911251]

10. Hart SG. Nasa-Task Load Index (NASA-TLX); 20 Years Later. Proceedings of the Human Factors and Ergonomics Society Annual Meeting 2016 Nov 05;50(9):904-908. [doi: 10.1177/154193120605000909]

11. Grier RA. How High is High? A Meta-Analysis of NASA-TLX Global Workload Scores. Proceedings of the Human Factors and Ergonomics Society Annual Meeting 2016 Dec 20;59(1):1727-1731. [doi: 10.1177/1541931215591373]

12. Dias RD, Ngo-Howard MC, Boskovski MT, Zenati MA, Yule SJ. Systematic review of measurement tools to assess surgeons' intraoperative cognitive workload. Br J Surg 2018 Apr;105(5):491-501 [FREE Full text] [doi: 10.1002/bjs.10795] [Medline: 29465749]

13. Peng X, Xu Z, Peng X. [Comparison of medical student's mental workload between VDT and paper-based reading]. Zhonghua Lao Dong Wei Sheng Zhi Ye Bing Za Zhi 2008 Dec;26(12):738-740. [Medline: 19257934]

14. Fournier LR, Wilson GF, Swain CR. Electrophysiological, behavioral, and subjective indexes of workload when performing multiple tasks: manipulations of task difficulty and training. Int J Psychophysiol 1999 Jan;31(2):129-145. [doi:

10.1016/s0167-8760(98)00049-x] [Medline: 9987059]

15. Qiao F, Zhang R, Yu L. Using NASA-Task Load Index to Assess Drivers' Workload on Freeway Guide Sign Structures. In: ICCTP 2011: Towards Sustainable Transportation Systems. Nanjing, China: ICCTP; 2011:4342-4353.

16. Hendy KC, Hamilton KM, Landry LN. Measuring Subjective Workload: When Is One Scale Better Than Many? Hum Factors 2016 Nov 23;35(4):579-601. [doi: 10.1177/001872089303500401]

17. Nygren TE. Psychometric Properties of Subjective Workload Measurement Techniques: Implications for Their Use in the Assessment of Perceived Mental Workload. Hum Factors 2016 Nov 23;33(1):17-33. [doi: 10.1177/001872089103300102]

18. Tscholl DW, Weiss M, Handschin L, Spahn DR, Nöthiger CB. User perceptions of avatar-based patient monitoring: a mixed qualitative and quantitative study. BMC Anesthesiol 2018 Dec 11;18(1):188 [FREE Full text] [doi: 10.1186/s12871-018-0650-1] [Medline: 30537934]

19. Kieseier BC, Bauer M. [Patient monitoring 4.0]. Nervenarzt 2019 Dec;90(12):1205-1206. [doi: 10.1007/s00115-019-00823-w] [Medline: 31807830]

20. Zimlichman E, Szyper-Kravitz M, Unterman A, Goldman A, Levkovich S, Shoenfeld Y. How is my patient doing? Evaluating hospitalized patients using continuous vital signs monitoring. Isr Med Assoc J 2009 Jun;11(6):382-384 [FREE Full text] [Medline: 19697594]

21. Tscholl DW, Handschin L, Neubauer P, Weiss M, Seifert B, Spahn DR, et al. Using an animated patient avatar to improve perception of vital sign information by anaesthesia professionals. Br J Anaesth 2018 Sep;121(3):662-671 [FREE Full text] [doi: 10.1016/j.bja.2018.04.024] [Medline: 30115265]

22. Tscholl DW, Rössler J, Said S, Kaserer A, Spahn DR, Nöthiger CB. Situation Awareness-Oriented Patient Monitoring with Visual Patient Technology: A Qualitative Review of the Primary Research. Sensors (Basel) 2020 Apr 09;20(7) [FREE Full text] [doi: 10.3390/s20072112] [Medline: 32283625]

23. Rössler J, Meybohm P, Spahn DR, Zacharowski K, Braun J, Nöthiger CB, et al. Improving decision making through presentation of viscoelastic tests as a 3D animated blood clot: the Visual Clot. Anaesthesia 2020 Aug;75(8):1059-1069. [doi: 10.1111/anae.14985] [Medline: 32030729]

24. Ruiz-Rabelo JF, Navarro-Rodriguez E, Di-Stasi LL, Diaz-Jimenez N, Cabrera-Bermon J, Diaz-Iglesias C, et al. Validation of the NASA-TLX Score in Ongoing Assessment of Mental Workload During a Laparoscopic Learning Curve in Bariatric Surgery. Obes Surg 2015 Dec;25(12):2451-2456. [doi: 10.1007/s11695-015-1922-1] [Medline: 26459432]

25. Tubbs-Cooley HL, Mara CA, Carle AC, Gurses AP. The NASA Task Load Index as a measure of overall workload among neonatal, paediatric and adult intensive care nurses. Intensive Crit Care Nurs 2018 Jun;46:64-69. [doi:

10.1016/j.iccn.2018.01.004] [Medline: 29449130] 
26. Miyake S. [Mental Workload Assessment of Health Care Staff by NASA-TLX]. J UOEH 2020;42(1):63-75 [FREE Full text] [doi: $\underline{10.7888 / j u o e h .42 .63}$ [ [Medline: $\underline{32213744]}$

27. Lowndes BR, Forsyth KL, Blocker RC, Dean PG, Truty MJ, Heller SF, et al. NASA-TLX Assessment of Surgeon Workload Variation Across Specialties. Ann Surg 2020 Apr;271(4):686-692. [doi: 10.1097/SLA.0000000000003058] [Medline: $\underline{30247331]}$

28. Pfarr J, Ganter MT, Spahn DR, Noethiger CB, Tscholl DW. Effects of a standardized distraction on caregivers' perceptive performance with avatar-based and conventional patient monitoring: a multicenter comparative study. J Clin Monit Comput 2019 Nov 25. [doi: 10.1007/s10877-019-00429-2] [Medline: 31768924]

29. Garot O, Rössler J, Pfarr J, Ganter MT, Spahn DR, Nöthiger CB, et al. Avatar-based versus conventional vital sign display in a central monitor for monitoring multiple patients: a multicenter computer-based laboratory study. BMC Med Inform Decis Mak 2020 Feb 10;20(1):26 [FREE Full text] [doi: 10.1186/s12911-020-1032-4] [Medline: 32041584]

30. Fink A. Survey Research Methods. In: Peterson P, Baker E, McGaw B, editors. International Encyclopedia of Education (Third Edition). Oxford: Elsevier; 2010:152-160.

31. Loft S, Sanderson P, Neal A, Mooij M. Modeling and predicting mental workload in en route air traffic control: critical review and broader implications. Hum Factors 2007 Jun;49(3):376-399. [doi: 10.1518/001872007X197017] [Medline: 17552304]

32. Mental AV, Pamela ST. Mental Workload and Situation Awareness. In: Handbook of Human Factors and Ergonomics. Hoboken, New Jersey: John Wiley \& Sons, Inc; 2012.

33. Wheelock A, Suliman A, Wharton R, Babu ED, Hull L, Vincent C, et al. The Impact of Operating Room Distractions on Stress, Workload, and Teamwork. Ann Surg 2015 Jun;261(6):1079-1084. [doi: 10.1097/SLA.0000000000001051] [Medline: 26291954]

34. Binenbaum G, Musick DW, Ross HM. The development of physician confidence during surgical and medical internship. Am J Surg 2007 Jan;193(1):79-85. [doi: 10.1016/j.amjsurg.2006.07.009] [Medline: 17188093]

35. Endsley M, Jones W. Situation awareness information dominance \& information warfare. In: Air Force Research Laboratory. Ohio: United States Airforce; 1997.

36. Burton J. WHO Healthy Workplace Framework and Model: Background and Supporting Literature and Practice. Geneva: World Health Organization; 2010.

37. Bowling NA, Alarcon GM, Bragg CB, Hartman MJ. A meta-analytic examination of the potential correlates and consequences of workload. Work \& Stress 2015 Apr 17;29(2):95-113. [doi: 10.1080/02678373.2015.1033037]

38. de Beer LT, Pienaar J, Rothmann S. Work overload, burnout, and psychological ill-health symptoms: a three-wave mediation model of the employee health impairment process. Anxiety Stress Coping 2016 Jul;29(4):387-399. [doi: 10.1080/10615806.2015.1061123] [Medline: 26079200]

39. Carthon JM, Kutney-Lee A, Jarrín O, Sloane D, Aiken LH. Nurse staffing and postsurgical outcomes in black adults. J Am Geriatr Soc 2012 Jun;60(6):1078-1084 [FREE Full text] [doi: 10.1111/j.1532-5415.2012.03990.x] [Medline: 22690984]

40. Wallace DJ, Angus DC, Barnato AE, Kramer AA, Kahn JM. Nighttime intensivist staffing and mortality among critically ill patients. N Engl J Med 2012 May 31;366(22):2093-2101 [FREE Full text] [doi: 10.1056/NEJMsa1201918] [Medline: 22612639]

41. Landrigan CP, Rothschild JM, Cronin JW, Kaushal R, Burdick E, Katz JT, et al. Effect of reducing interns' work hours on serious medical errors in intensive care units. N Engl J Med 2004 Oct 28;351(18):1838-1848. [doi: 10.1056/NEJMoa041406] [Medline: 15509817]

42. Grier RA, Warm JS, Dember WN, Matthews G, Galinsky TL, Parasuraman R. The vigilance decrement reflects limitations in effortful attention, not mindlessness. Hum Factors 2003;45(3):349-359. [doi: 10.1518/hfes.45.3.349.27253] [Medline: 14702988]

43. Broom MA, Capek AL, Carachi P, Akeroyd MA, Hilditch G. Critical phase distractions in anaesthesia and the sterile cockpit concept. Anaesthesia 2011 Mar;66(3):175-179 [FREE Full text] [doi: 10.1111/j.1365-2044.2011.06623.x] [Medline: 21320085]

44. Schulz CM, Burden A, Posner KL, Mincer SL, Steadman R, Wagner KJ, et al. Frequency and Type of Situational Awareness Errors Contributing to Death and Brain Damage: A Closed Claims Analysis. Anesthesiology 2017 Aug;127(2):326-337 [FREE Full text] [doi: 10.1097/ALN.0000000000001661] [Medline: 28459735]

45. Crockett CJ, Donahue BS, Vandivier DC. Distraction-Free Induction Zone: A Quality Improvement Initiative at a Large Academic Children's Hospital to Improve the Quality and Safety of Anesthetic Care for Our Patients. Anesth Analg 2019 Sep;129(3):794-803. [doi: 10.1213/ANE.0000000000003879] [Medline: 31425222]

46. Tscholl DW, Handschin L, Rössler J, Weiss M, Spahn DR, Nöthiger CB. It's not you, it's the design - common problems with patient monitoring reported by anesthesiologists: a mixed qualitative and quantitative study. BMC Anesthesiol 2019 May 28;19(1):87 [FREE Full text] [doi: 10.1186/s12871-019-0757-z] [Medline: 31138143]

47. McNeer RR, Bennett CL, Dudaryk R. Intraoperative Noise Increases Perceived Task Load and Fatigue in Anesthesiology Residents: A Simulation-Based Study. Anesth Analg 2016 Feb;122(2):512-525. [doi: 10.1213/ANE.0000000000001067] [Medline: 26797555] 
48. Yurko YY, Scerbo MW, Prabhu AS, Acker CE, Stefanidis D. Higher mental workload is associated with poorer laparoscopic performance as measured by the NASA-TLX tool. Simul Healthc 2010 Oct;5(5):267-271. [doi: 10.1097/SIH.0b013e3181e3f329] [Medline: 21330808]

49. Hu JS, Lu J, Tan WB, Lomanto D. Training improves laparoscopic tasks performance and decreases operator workload. Surg Endosc 2016 May;30(5):1742-1746. [doi: 10.1007/s00464-015-4410-8] [Medline: 26173550]

50. Finomore VS, Shaw TH, Warm JS, Matthews G, Boles DB. Viewing the workload of vigilance through the lenses of the NASA-TLX and the MRQ. Hum Factors 2013 Dec;55(6):1044-1063. [doi: 10.1177/0018720813484498] [Medline: 24745198]

51. Rubio-Valdehita S, López-Núñez MI, López-Higes R, Díaz-Ramiro EM. Development of the CarMen-Q Questionnaire for mental workload assessment. Psicothema 2017 Nov;29(4):570-576. [doi: 10.7334/psicothema2017.151] [Medline: $\underline{29048320}$ ]

52. Zheng B, Jiang X, Tien G, Meneghetti A, Panton ON, Atkins MS. Workload assessment of surgeons: correlation between NASA TLX and blinks. Surg Endosc 2012 Oct;26(10):2746-2750. [doi: 10.1007/s00464-012-2268-6] [Medline: 22527300]

53. Marinescu AC, Sharples S, Ritchie AC, Sánchez López T, McDowell M, Morvan HP. Physiological Parameter Response to Variation of Mental Workload. Hum Factors 2018 Feb;60(1):31-56 [FREE Full text] [doi: 10.1177/0018720817733101] [Medline: 28965433]

\section{Abbreviations \\ NASA-TLX: National Aeronautics and Space Administration-Task Load Index \\ ROTEM: rotational thromboelastometry}

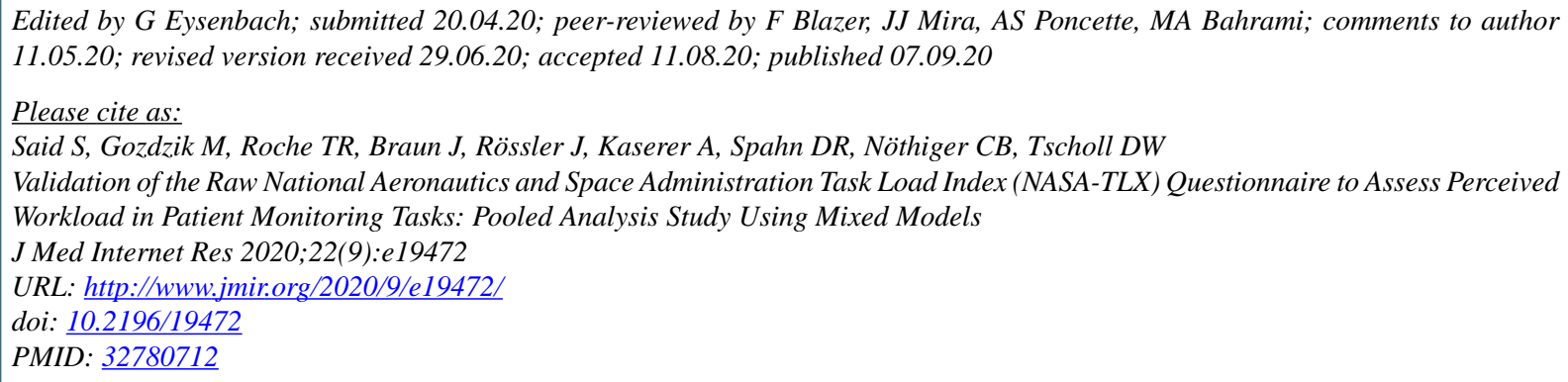

(C) Sadiq Said, Malgorzata Gozdzik, Tadzio Raoul Roche, Julia Braun, Julian Rössler, Alexander Kaserer, Donat R Spahn, Christoph B Nöthiger, David Werner Tscholl. Originally published in the Journal of Medical Internet Research (http://www.jmir.org), 07.09.2020. This is an open-access article distributed under the terms of the Creative Commons Attribution License (https://creativecommons.org/licenses/by/4.0/), which permits unrestricted use, distribution, and reproduction in any medium, provided the original work, first published in the Journal of Medical Internet Research, is properly cited. The complete bibliographic information, a link to the original publication on http://www.jmir.org/, as well as this copyright and license information must be included. 